\title{
How cardiologists manage antithrombotic treatment of patients with atrial fibrillation undergoing percutaneous coronary stenting: the WOEST survey 2018
}

\author{
A. J. W. M. de Veer · N. Bennaghmouch · W. J. M. Dewilde · J. M. ten Berg
}

Accepted: 30 September 2020 / Published online: 14 October 2020

(c) The Author(s) 2020

\begin{abstract}
Background Antithrombotic treatment choices are complicated when patients have both atrial fibrillation (AF) and acute coronary syndrome and/or undergo percutaneous coronary intervention (PCI). In this study, we aimed to gain insight into antithrombotic management strategies in daily clinical practice. Methods We invited interventional cardiologists to complete the WOEST (What is the Optimal antiplatElet \& Anticoagulant Therapy in Patients With Oral Anticoagulation and Coronary StenTing) survey 2018. In this questionnaire, we presented a patient with a non-ST-elevation myocardial infarction (NSTEMI) and an elective PCI case.

Results The results were based on 118 completed questionnaires (response rate $69.4 \%$ ). In the case of the AF patient with NSTEMI, most cardiologists indicated they would initiate dual antiplatelet therapy (acetylsalicylic acid and clopidogrel) and continue non-vitamin $\mathrm{K}$ antagonist oral anticoagulant (NOAC) therapy at admission and during coronary angiography/PCI. At discharge, $70.3 \%$ would prescribe triple antithrombotic therapy (oral anticoagulation, acetylsalicylic acid and clopidogrel), mostly for 1 month. One year after NSTEMI, 83.1\% would cancel the antiplatelet therapy and prescribe NOAC monotherapy.
\end{abstract}

Electronic supplementary material The online version of this article (https://doi.org/10.1007/s12471-020-01500-3) contains supplementary material, which is available to authorized users.

A. J. W. M. de Veer $(\bowtie)$ N N. Bennaghmouch $\cdot$ J. M. ten Berg Department of Cardiology, St. Antonius Hospital,

Nieuwegein, The Netherlands

annedeveer@outlook.com

W. J. M. Dewilde

Department of Cardiology, Imelda Hospital, Bonheiden, Belgium
For the AF patient undergoing elective PCI, $51.7 \%$ would start dual antiplatelet therapy prior to the procedure and $52.5 \%$ would discontinue NOAC therapy prior to the PCI. At discharge, $55.1 \%$ would start triple antithrombotic therapy. Furthermore, 25.4\% responded they routinely prescribe a reduced dose of NOAC after discharge. One year after PCI, 89.0\% would continue NOAC monotherapy.

Conclusion The WOEST survey demonstrated heterogeneity in antithrombotic management strategies among interventional cardiologists. This observed variety mirrors the heterogeneity of the many guidelines and consensus documents. Further research is needed to guide patient-tailored medicine for AF patients undergoing PCI.

Keywords Anticoagulation - Antiplatelet therapy • Acute coronary syndrome $\cdot$ Percutaneous coronary intervention · Atrial fibrillation · Non-vitamin K antagonist oral anticoagulants

What's new?

- To investigate antithrombotic management strategies for atrial fibrillation (AF) patients treated with oral anticoagulation undergoing percutaneous coronary intervention (PCI) with stenting, we carried out a survey among interventional cardiologists.

- Dual antiplatelet therapy was the most prescribed antithrombotic regimen for AF patients admitted to the hospital with non-ST-elevation myocardial infarction.

- One year after PCI, most of the cardiologists (>80\%) would cancel the antiplatelet therapy and continue monotherapy with a non-vitamin $\mathrm{K}$ antagonist oral anticoagulant. 


\section{Introduction}

For patients with atrial fibrillation (AF) and an increased $\mathrm{CHA}_{2} \mathrm{DS}_{2}$-VASc score $(\geq 2$ for men and $\geq 3$ for women), guidelines recommend lifelong treatment with oral anticoagulation (OAC) to reduce stroke risk [1]. For patients with acute coronary syndrome (ACS) or undergoing percutaneous coronary intervention (PCI) with stenting, guidelines recommend treatment with dual antiplatelet therapy (DAPT), consisting of acetylsalicylic acid and a $\mathrm{P}_{2} \mathrm{Y}_{12}$ inhibitor to reduce the risk of stent thrombosis and other atherothrombotic events [2, 3]. However, treatment choices are complicated when patients have both AF and ACS and/or undergo PCI. Current clinical guidelines acknowledge considerable gaps in the availability of high-quality evidence regarding optimal antithrombotic therapy for these patients.

Recently, the results of four randomised controlled trials have been published, in which the effect of vitamin $\mathrm{K}$ antagonists (VKAs) versus non-vitamin $\mathrm{K}$ antagonist oral anticoagulants (NOACs) in combination with antiplatelet therapy was investigated in patients undergoing PCI with stenting [4-7]. Furthermore, European and American expert consensus documents have been updated $[8,9]$. According to the European guidelines, most patients should be treated with both OAC and DAPT (i.e. triple antithrombotic therapy). However, the inevitable trade-off of intensification of antithrombotic therapy are more bleeding complications, which is associated with increased mortality [10-12]. Therefore, antithrombotic treatment for this complex population should be carefully balanced.

We have noticed important differences in antithrombotic strategies carried out by cardiologists. To obtain insight into the current antithrombotic strategies in daily clinical practice for patients with both AF and ACS and/or undergoing PCI with stenting, we carried out a survey in Dutch and some international PCI centres.

\section{Methods}

The WOEST (What is the Optimal antiplatElet \& Anticoagulant Therapy in Patients With Oral Anticoagulation and Coronary StenTing) survey 2018 is a questionnaire addressing the antithrombotic treatment of patients with AF undergoing PCI with stenting in daily practice from the perspective of the treating interventional cardiologist. This survey was based on a 19-item online questionnaire sent out to all interventional cardiologists working in the 27 PCI centres in the Netherlands and to individual interventional cardiologists in 24 PCI centres in Belgium, Germany, the United Kingdom, Denmark, Austria, Switzerland, France, Spain, Italy, Greece, Portugal and Sweden. The survey was conducted from September to December 2018.
As an introduction to the questions posed, we presented two cases: (1) AF patient using a NOAC who is hospitalised for a non-ST-elevation myocardial infarction (NSTEMI) and needs urgent PCI, and (2) AF patient using a NOAC who is diagnosed with chronic coronary syndrome and needs to undergo elective PCI. Both patients carry an intermediate bleeding and thrombotic risk. A complete overview of the survey questions is shown in Tab. 1 (see Electronic Supplementary Material). The survey was reviewed by an expert panel.

The Medical research Ethics Committees United confirmed that the Medical Research Involving Human Subjects Act (Wet medisch-wetenschappelijk onderzoek met mensen) did not apply to this noninterventional study; hence, official ethical approval by the ethics committee was not required. Declaration approval was obtained from the Institutional Review Board. A local study protocol is available. The study was conducted in accordance with the Declaration of Helsinki.

\section{Statistical analysis}

Descriptive analysis of the data was performed using summary statistics for categorical and quantitative (continuous) data. Categorical data are expressed as frequencies with percentages. Statistical analysis was performed using SPSS software for Windows, version 24 (IBM Corporation, Armonk, NY, USA).

\section{Results}

\section{Participants}

A total of 118 interventional cardiologists completed the questionnaire (response rate $69.4 \%$ ). Of them, 100 cardiologists $(84.7 \%)$ worked in a total of 27 different PCI centres in the Netherlands.

\section{Outcomes}

Of the responding cardiologists, $73.7 \%$ reported that a standard local protocol is available for the antithrombotic management of patients on OAC who require $\mathrm{PCI}$.

For the AF patient with NSTEMI, 95.8\% of the cardiologists would start antiplatelet therapy as soon as possible after admission. The majority of these cardiologists $(70.3 \%)$ responded they initiate DAPT (acetylsalicylic acid and clopidogrel), 22.9\% initiate $\mathrm{P}_{2} \mathrm{Y}_{12}$ inhibitor monotherapy (ticagrelor $90 \mathrm{mg}$ twice daily, clopidogrel $75 \mathrm{mg}$ once daily or prasugrel $10 \mathrm{mg}$ once daily), and $6.8 \%$ initiate acetylsalicylic acid monotherapy. More than half $(56.8 \%)$ would continue NOAC therapy at admission and during coronary angiography/PCI. During PCI, 33.9\% would administer a reduced dose of heparin and $3.4 \%$ chose not to administer heparin at all. 
Table 1 Management of AF patients on NOAC therapy undergoing PCI, according to the WOEST survey 2018 among 118 cardiologists

\begin{tabular}{|c|c|c|c|}
\hline \multirow{2}{*}{\multicolumn{4}{|c|}{$\begin{array}{l}\text { Question } \\
\text { General }\end{array}$}} \\
\hline & & & \\
\hline \multirow{3}{*}{$\begin{array}{l}\text { Is there a standardised protocol for the antithrombotic management of } \\
\text { AF patients on VKA or NOAC who require PCI? }\end{array}$} & Yes, but only for patients VKA & \multicolumn{2}{|l|}{$4(3.4)$} \\
\hline & Yes, for patients on chronic VKA and NOAC & \multicolumn{2}{|l|}{$87(73.7)$} \\
\hline & No, there is no protocol & \multicolumn{2}{|l|}{$27(22.9)$} \\
\hline \multicolumn{2}{|l|}{ Before PCl } & NSTEMI & Elective $\mathrm{PCl}$ \\
\hline \multirow[t]{2}{*}{ Would you initiate antiplatelet therapy immediately after admission? } & Yes & $113(95.8)$ & $N / A$ \\
\hline & No, I would start this during/after $\mathrm{PCl}$ & $5(4.2)$ & $\mathrm{N} / \mathrm{A}$ \\
\hline \multirow[t]{3}{*}{ If yes, which antithrombotic regimen would you start? } & ASA monotherapy (+NOAC) & $8(6.8)$ & $\mathrm{N} / \mathrm{A}$ \\
\hline & $\mathrm{P} 2 \mathrm{Y}_{12}$ inhibitor monotherapy (+NOAC) & $27(22.9)$ & $\mathrm{N} / \mathrm{A}$ \\
\hline & Dual antiplatelet therapy (+NOAC) & $83(70.3)$ & $\mathrm{N} / \mathrm{A}$ \\
\hline \multirow[t]{3}{*}{ Would you discontinue the NOAC therapy before PCl? } & Yes, without bridging & $43(36.4)$ & $62(52.5)$ \\
\hline & Yes, with bridging & $8(6.8)$ & 0 \\
\hline & No & $67(56.8)$ & $56(47.5)$ \\
\hline \multicolumn{2}{|l|}{ During PCl } & NSTEMI & Elective PCl \\
\hline \multirow{3}{*}{$\begin{array}{l}\text { Would you administer a bolus of heparin (UFH/LMWH) at the start of PCl } \\
\text { when the patient is on NOAC? }\end{array}$} & Yes, the standard dose & $74(62.7)$ & $78(66.1)$ \\
\hline & Yes, a reduced dose & $30(33.9)$ & $35(29.7)$ \\
\hline & No & $4(3.4)$ & $5(4.2)$ \\
\hline \multicolumn{2}{|l|}{ After $\mathrm{PCl}$} & NSTEMI & Elective PCl \\
\hline \multirow{2}{*}{$\begin{array}{l}\text { At discharge, what is your default antithrombotic strategy for a patient } \\
\text { like this (intermediate bleeding risk and intermediate thrombotic risk)? }\end{array}$} & Triple therapy & $83(70.3)$ & $65(55.1)$ \\
\hline & Dual therapy & $35(29.7)$ & $53(44.9)$ \\
\hline \multirow{2}{*}{$\begin{array}{l}\text { In case of a high bleeding risk, what is your default antithrombotic } \\
\text { strategy at discharge? }\end{array}$} & Triple therapy & $11(9.3)$ & $6(5.1)$ \\
\hline & Dual therapy & $107(90.7)$ & $112(94.9)$ \\
\hline \multirow{2}{*}{$\begin{array}{l}\text { In case of a high bleeding risk and a high thrombotic risk, what is your } \\
\text { default antithrombotic strategy at discharge? }\end{array}$} & Triple therapy & $51(43.2)$ & 37 (31.4) \\
\hline & Dual therapy & $67(56.8)$ & $81(68.6)$ \\
\hline \multirow{5}{*}{$\begin{array}{l}\text { When you use triple therapy, for what period of time would you } \\
\text { prescribe ASA? }\end{array}$} & 1 month & $98(83.1)$ & $106(89.8)$ \\
\hline & 3 months & $8(6.8)$ & $4(3.4)$ \\
\hline & 6 months & $7(5.9)$ & $3(2.5)$ \\
\hline & 12 months & $4(3.4)$ & $3(2.5)$ \\
\hline & $>12$ months & $1(0.8)$ & $2(1.7)$ \\
\hline \multirow[t]{5}{*}{ Which NOAC for stroke prevention would you prescribe at discharge? } & I would continue the same NOAC & $93(78.8)$ & $97(82.2)$ \\
\hline & Dabigatran & $10(8.5)$ & $10(8.5)$ \\
\hline & Rivaroxaban & $2(1.7)$ & $1(0.8)$ \\
\hline & Apixaban & $11(9.3)$ & $8(6.8)$ \\
\hline & Edoxaban & $2(1.7)$ & $2(1.7)$ \\
\hline \multirow[t]{2}{*}{ Which dose would you prescribe at discharge? } & The normal dose & $77(65.3)$ & $88(74.6)$ \\
\hline & The reduced dose & $41(34.7)$ & $30(25.4)$ \\
\hline \multirow{2}{*}{$\begin{array}{l}\text { If the patient was using a VKA instead of a NOAC, would you switch the } \\
\text { patient to a NOAC after PCI? }\end{array}$} & Yes & $44(37.3)$ & $45(38.1)$ \\
\hline & No & $74(62.7)$ & $73(61.9)$ \\
\hline \multirow[t]{2}{*}{ Which antithrombotic treatment would you prescribe $>1$ year after $\mathrm{PCl}$ ? } & Monotherapy: NOAC & $98(83.1)$ & $105(89.0)$ \\
\hline & Dual therapy: NOAC and ASA or $\mathrm{P}_{2} \mathrm{Y}_{12}$ inhibitor & $20(16.9)$ & $2(11.0)$ \\
\hline
\end{tabular}

$A F$ atrial fibrillation, NOAC non-vitamin $\mathrm{K}$ antagonist oral anticoagulant, $P C /$ percutaneous coronary intervention, VKA vitamin $\mathrm{K}$ antagonist, NSTEMI non-ST-elevation myocardial infarction, $A S A$ acetylsalicylic acid, UFH/LMWH unfractionated heparin/low-molecular weight heparin, N/A not applicable

At discharge, most of the cardiologists (70.3\%) would treat a patient with an intermediate bleeding risk as well as an intermediate thrombotic risk with triple antithrombotic therapy. In case of a high bleeding risk, $9.3 \%$ would start triple therapy; in case of both a high bleeding and a high thrombotic risk, $43.2 \%$ would initiate this treatment. When prescribing triple antithrombotic therapy, the majority
(83.1\%) would do this for 1 month. A reduced dose of a NOAC (rivaroxaban $15 \mathrm{mg}$ once daily, apixaban $2.5 \mathrm{mg}$ twice daily, dabigatran $110 \mathrm{mg}$ twice daily in patients $<80$ years, edoxaban $30 \mathrm{mg}$ once daily) would be prescribed by $34.7 \%$ of the cardiologists; the others would prescribe the routinely dosed NOAC. After 1 year, the majority $(83.1 \%)$ would cancel the antiplatelet therapy and prescribe NOAC monotherapy. 
For the AF patient undergoing elective PCI, 51.7\% would start DAPT prior to elective PCI. The others would start single antiplatelet therapy, mainly a $\mathrm{P}_{2} \mathrm{Y}_{12}$ inhibitor. More than half $(52.5 \%)$ indicated to discontinue NOAC therapy prior to PCI and no one chose to bridge the anticoagulation therapy. If NOAC therapy is continued during PCI, $4.2 \%$ would not administer heparin during the procedure, which means that 95.8\% would give heparin, one-third of whom would use a reduced dose.

At discharge, the default strategy was triple antithrombotic therapy for more than half of the cardiologists (55.1\%); the others would start dual antithrombotic therapy consisting of a $\mathrm{P}_{2} \mathrm{Y}_{12}$ inhibitor and an oral anticoagulant. In case of a high bleeding risk, $5.1 \%$ would start triple antithrombotic therapy; in case of both a high bleeding and a high thrombotic risk, $31.4 \%$ would start this treatment. When triple antithrombotic therapy is prescribed, $89.8 \%$ would prescribe acetylsalicylic acid for 1 month. Furthermore, $25.4 \%$ indicated to prescribe a reduced dose of NOAC after discharge. One year after PCI, 89.0\% would stop the antiplatelet therapy and continue NOAC monotherapy.

In the elective PCI setting, $38.1 \%$ of the cardiologists would switch to a NOAC if the patient is using a VKA. If the patient has ACS, is using a VKA and is in urgent need of PCI, $37.3 \%$ would switch to a NOAC. All results are shown in Tab. 1.

\section{Discussion}

The WOEST survey 2018 provides insight into daily clinical practice with regard to antithrombotic therapy in AF patients with ACS and/or undergoing PCI in European PCI centres. We observed a large heterogeneity in management strategies among the interventional cardiologists.

The recent guidelines from the European Society of Cardiology (ESC) on the management of patients with AF favour the use of NOACs over VKAs in patients with nonvalvular AF [1]. As approximately $20 \%$ of AF patients require PCI at some stage, many patients need additional antiplatelet therapy [13]. However, there is currently no clear guideline on the management of NOACs in patients undergoing PCI, similar to when these patients are using a VKA. This could explain why $>40 \%$ of the cardiologists in our survey would discontinue NOAC therapy in patients with NSTEMI undergoing PCI. In line with this observation, $53 \%$ of the cardiologists would discontinue the NOAC before performing an elective PCI. This indicates a reluctance to treat patients with PCI when they are on NOAC therapy.

This reluctance might be explained by the lack of evidence for the safety of NOACs in the catheterisation laboratory and the heterogeneity in practice guideline recommendations for these patients. In a small randomised phase IIa study, more thrombotic events occurred in patients treated with dabigatran (direct thrombin inhibitor) during elective PCI than in those treated with unfractionated heparin [14]. As for rivaroxaban (direct factor Xa inhibitor), the X-PLORER study has shown that rivaroxaban (with or without unfractionated heparin) effectively inhibits coagulation activity during elective PCI in patients with chronic coronary syndrome compared with unfractionated heparin alone [15]. Furthermore, the OASIS-5 trial has shown that the use of fondaparinux (factor Xa inhibitor) results in more catheter-related thrombosis than treatment with enoxaparin (lowmolecular weight heparin) [16]. This may be one of the reasons why some interventional cardiologists are less confident in the performance of NOACs in the catheterisation laboratory.

The small WOEST trial was the first to compare the combination of a VKA plus clopidogrel to conventional triple therapy (consisting of VKA, clopidogrel and acetylsalicylic acid) and has shown that cancelling acetylsalicylic acid decreases the incidence of bleeding events in AF patients undergoing PCI without an increase in the incidence of thromboembolic events [17]. Following the WOEST trial, four international randomised trials have been conducted investigating the role of a NOAC-based antithrombotic strategy versus conventional triple therapy. In the PIONEER AF-PCI trial (A Study Exploring Two Strategies of Rivaroxaban and One of Oral Vitamin K Antagonist in Patients With Atrial Fibrillation Who Undergo Percutaneous Coronary Intervention), two rivaroxaban-based treatment regimens significantly reduced bleeding complications following PCI compared with conventional triple therapy, without increasing thromboembolic complications [4] Of note, the tested rivaroxaban dose (15 $\mathrm{mg}$ once daily) differed from the dose used in the AF trial $(20 \mathrm{mg}$ once daily). Dual therapy with dabigatran also reduces bleeding complications, as shown in the REDUAL-AF PCI trial (Evaluation of Dual Therapy With Dabigatran vs. Triple Therapy With Warfarin in Patients With AF That Undergo a PCI With Stenting) [5].

The AUGUSTUS trial (An Open-label, $2 \times 2$ Factorial, Randomised Controlled, Clinical Trial to Evaluate the Safety of Apixaban vs. Vitamin K Antagonist and Aspirin vs. Aspirin Placebo in Patients with Atrial Fibrillation and Acute Coronary Syndrome or Percutaneous Coronary Intervention) is the only trial that compared NOAC versus VKA plus DAPT and NOAC versus VKA with single antiplatelet therapy in a $2 \times 2$ design. It has shown that acetylsalicylic acid (aspirin), compared with placebo, is associated with significantly more bleeding events, but that the use of apixaban, compared with VKA, reduces the number of bleeding events [6]. Furthermore, in the ENTRUSTAF PCI trial (Edoxaban Treatment Versus Vitamin K Antagonist in Patients With Atrial Fibrillation Undergoing Percutaneous Coronary Intervention), edoxaban dual antithrombotic therapy was noninferior to 
triple antithrombotic therapy with respect to major or clinically relevant nonmajor bleeding [7].

Finally, recent meta-analyses have shown that in patients with AF undergoing PCI, dual antithrombotic therapy (either NOAC or VKA in combination with single antiplatelet therapy) reduces the composite of a TIMI (thrombolysis in myocardial infarction) major or minor bleeding by $47 \%$ as well as intracranial haemorrhages $[18,19]$. Although the four trials were not powered to detect differences in thromboembolic events [4-7]; in none of the studies, this endpoint was significantly increased in the patients treated with dual therapy.

Evaluating all available data, one might argue that most evidence points to a NOAC-based regimen of dual therapy. Conversely, the current ESC guidelines (last updated before the AUGUSTUS and ENTRUST trials) recommend dual therapy only for selected patients with a (very) high risk of bleeding. It is, however, difficult to define patients with a high bleeding risk. Although there are several scores to assess bleeding risk (HAS-BLED for AF patients, CRUSADE for ACS patients, PRECISE-DAPT for PCI patients) [20-22], none is specifically suitable for AF patients undergoing PCI. On the other hand, there may be patients with a very high thrombotic risk for whom a longer period of triple therapy ( $>1$ month) is advised by the current ESC guidelines. Similar to the bleeding risk, the thrombotic risk is difficult to define. It is also not clear how the currently available thromboembolic risk scores $\left(\mathrm{CHA}_{2} \mathrm{DS}_{2}\right.$-VASc score and DAPT score) perform in these patients. Nevertheless, the data call for more patient-tailored antithrombotic management [23, 24].

For patients with chronic coronary syndrome and NSTEMI, roughly two-thirds of the interventional cardiologists would prescribe a full NOAC dose when the NOAC is combined with single or dual antiplatelet therapy. The practical guide on the use of NOACs suggests reducing the NOAC dose in case of a high bleeding risk in patients with concomitant antiplatelet therapy [25]. However, only dabigatran $110 \mathrm{mg}$ twice daily has shown to be effective in stroke prevention; the reduced doses of other NOACs (rivaroxaban $15 \mathrm{mg}$ once daily, apixaban $2.5 \mathrm{mg}$ twice daily, edoxaban $30 \mathrm{mg}$ once daily) have not. Hence, lowering the dose of NOACs might result in more thromboembolic events. In a recently published retrospective analysis, off-label use of the reduced dose of NOACs was associated with a 2.5 times increased risk of thromboembolism compared with warfarin [26].

A few studies have investigated the combination of a NOAC and antiplatelet therapy in patients with acute or chronic coronary syndrome without AF. In the APPRAISE-2 trial, patients with recent ACS (median time 6 days) were randomly assigned to apixaban $5 \mathrm{mg}$ twice daily on top of dual antiplatelet therapy or antiplatelet therapy alone. The addition of apixaban results in significantly more bleeding events, without less recurrent ischaemic events [27]. Although low- dose rivaroxaban (2.5 or $5 \mathrm{mg}$ twice daily) on top of antiplatelet therapy leads to more bleeding in patients with ACS, this is counterbalanced by less cardiovascular events, including mortality [28]. In the large COMPASS trial studying patients with stable coronary artery disease, low-dose rivaroxaban ( $5 \mathrm{mg}$ twice daily) plus acetylsalicylic acid resulted in an absolute risk reduction in cardiovascular death, stroke or nonfatal myocardial infarction, at the expense of more bleeding. The net clinical benefit was in favour of rivaroxaban on top of acetylsalicylic acid [29]. However, in the previously mentioned trials the studied NOAC doses were lower than those approved for stroke prevention in AF patients.

The most recent evidence comes from the AFIRE study. In this trial, patients with AF and chronic coronary syndrome who had undergone PCI or coronary artery bypass grafting at least 1 year before enrolment were randomised to rivaroxaban monotherapy $(5 \mathrm{mg}$ twice daily) or rivaroxaban (5mg twice daily) plus a platelet inhibitor. Rivaroxaban monotherapy was noninferior to combination therapy for the composite primary endpoint of stroke, systemic embolism, myocardial infarction, unstable angina requiring revascularisation or death from any cause (hazard ratio 0.72, 95\% confidence interval [CI] 0.55-0.95) and superior in terms of major bleeding according to the criteria of the International Society on Thrombosis and Haemostasis (hazard ratio 0.59, 95\% CI 0.39-0.89) [30]. In our survey, the majority of the interventional cardiologists $(>80 \%)$ would prescribe NOAC monotherapy 1 year after PCI.

\section{Study limitations}

Our study has some limitations. Despite the high response rate $(69.4 \%)$, nonresponders might reflect those with less interest in and knowledge of the topic (response bias). Furthermore, we did not invite all European interventional cardiologists, but only a selection. Therefore, this survey is representative of current clinical practice in the Netherlands, but not across Europe. Finally, as in most surveys, the study relied on self-report and we did not validate self-report against objective measures.

\section{Conclusion}

In this online, international survey among interventional cardiologists, we observed heterogeneity in antithrombotic management strategies for AF patients treated with OAC undergoing PCI. This observed variety mirrors the heterogeneity of the many guidelines and consensus documents. Further research is needed to guide patient-tailored medicine for AF patients undergoing PCI.

Conflict of interest A.J.W.M. de Veer received speaker's and/or consulting fees from Bayer and Boehringer Ingelheim. N. Ben- 
naghmouch has nothing to declare. W.J.M. Dewilde received speaker's fees from Bayer and Daiichi Sankyo. J.M. ten Berg reports advisory/consulting/speaker's fees from Accumetrics, AstraZeneca, Bayer, Boehringer Ingelheim, Bristol-Myers Squibb, Daiichi Sankyo, Eli Lilly, The Medicines Company, Pfizer, Ferrer, and institutional research grants from AstraZeneca and ZonMw.

Open Access This article is licensed under a Creative Commons Attribution 4.0 International License, which permits use, sharing, adaptation, distribution and reproduction in any medium or format, as long as you give appropriate credit to the original author(s) and the source, provide a link to the Creative Commons licence, and indicate if changes were made. The images or other third party material in this article are included in the article's Creative Commons licence, unless indicated otherwise in a credit line to the material. If material is not included in the article's Creative Commons licence and your intended use is not permitted by statutory regulation or exceeds the permitted use, you will need to obtain permission directly from the copyright holder. To view a copy of this licence, visit http://creativecommons.org/licenses/by/4.0/.

\section{References}

1. Kirchhof P, Benussi S, Kotecha D, et al. 2016 ESC guidelines for the management of atrial fibrillation developed in collaboration with EACTS: the task force for the management of atrial fibrillation of the European society of cardiology (ESC). Developed with the special contribution of the European heart rhythm association (EHRA) of the ESC. Endorsed by the European stroke organisation (ESO). Eur Heart J.2016;37:2893-962.

2. Roffi M, Patrono C, Collet JP, et al. 2015 ESC guidelines for the management of acute coronary syndromes in patients presenting without persistent ST-segment elevation: task force for the management of acute coronary syndromes in patients presenting without persistent ST-segment elevation of the European society of cardiology (ESC). Eur Heart J. 2016;37:267-315.

3. Sousa-Uva M, Ahlsson A, Alfonso F, et al. 2018 ESC/ EACTS guidelines on myocardial revascularization the task force on myocardial revascularization of the European society of cardiology (ESC) and European association for Cardio-thoracic surgery (EACTS) developed with the special contribution of the European association for percutaneous cardiovascular interventions (EAPCI). Eur Heart J. 2018;00:1-96.

4. Gibson CM, Mehran R, BodeC, et al. Prevention of bleeding in patients with atrial fibrillation undergoing PCI. N Engl J Med. 2016;375:2423-34.

5. Cannon CP, Bhatt DL, Oldgren J, RE-DUAL PCI Steering Committee and Investigators, et al. Dual antithrombotic therapywith dabigatran after PCIin atrialfibrillation. NEngl J Med. 2017;377:1513-24.

6. Lopes RD, Heizer G, Aronson R, et al. Antithrombotic therapy after acute coronary syndrome or PCI in atrial fibrillation. NEngl J Med. 2019;380:1509-24.

7. Vrankx P, Valgimigli M, Eckardt L, et al. Edoxaban-based versus vitamin $K$ antagonist based antithrombotic regimen after succesful coronary stenting in patients with atrial fibrillation (ENTRUST AF-PCI): a randomised, open-label, phase 3b trial. Lancet. 2019;394:1335-43.

8. Lip GYH, Windecker S, Huber K, et al. Management of antithrombotic therapy in atrial fibrillation patients presenting with acute coronary syndrome and/or undergoing percutaneous coronary or valve interventions: a joint con- sensus document of the European society of cardiology working group on thrombosis, European heart rhythm association (EHRA), European association of percutaneous cardiovascular interventions (EAPCI) and European association of acute cardiac care (ACCA) endorsed by the heart rhythm society (HRS) and asia-pacific heart rhythm society (APHRS).Europace. 2019;21:192-3.

9. Angiolillo DJ, Goodman SG, Bhatt DL, et al. Antithrombotic therapy in patients with atrial fibrillation treated with oral anticoagulation undergoing percutaneous coronary intervention: a North American perspective-2018 update. Circulation. 2018;138:527-36.

10. Lamberts M, Olesen JB, Ruwald MH, et al. Bleeding after initiation of multiple antithrombotic drugs, including triple therapy, in atrial fibrillation patients following myocardial infarction and coronary intervention: a nationwide cohort study. Circulation. 2012;126:1185-93.

11. Hansen ML, Sorensen R, Clausen MT, et al. Risk of bleeding with single, dual, or triple therapy with warfarin, aspirin, and clopidogrel in patients with atrial fibrillation. Arch Intern Med. 2010;170:1433-41.

12. Manoukian SV, Feit F, Mehran R, et al. Impact of major bleeding on 30-day mortality and clinical outcomes in patients with acute coronary syndromes: an analysis from the ACUITY trial. JAm Coll Cardiol. 2007;49:1362-8.

13. Depta JP, Bhatt DL. Atherothrombosis and atrial fibrillation: important and often overlapping clinical syndromes. Thromb Haemost. 2010;104:657-63.

14. Vranckx P, Verheugt FW, de Maat MP, et al. A randomised study of dabigatran in elective percutaneous coronary intervention in stable coronary artery disease patients. EuroIntervention. 2013;8:1052-60.

15. Vranckx P, LeebeekFW, Tijssen JG, et al. Peri-procedural use of rivaroxaban in elective percutaneous coronary intervention to treat stable coronary artery disease. The XPLORER trial. Thromb Haemost. 2015;114:258-67.

16. Yusuf S, Mehta SR, Chrolavicius S, et al. Comparison of fondaparinux and enoxaparin in acute coronary syndromes. NEngJ Med. 2006;354:1464-76.

17. Dewilde WJM, Oirbans T, Verheugt FW, et al. Use of clopidogrel with or without aspirin in patients taking oral anticoagulant therapy and undergoing percutaneous coronary intervention: an open-label, randomised, controlled trial. Lancet. 2013;381:1107-15.

18. Golwala H, Cannon C, Steg Ph, et al. Safety and efficacy of dual vs. triple antithrombotic therapy in patients with atrial fibrillation following percutaneous coronary intervention: a systematic review and meta-analysis of randomized clinical trials. Eur Heart J. 2018;39:1726-135a.

19. Gargiulo G, Goette A, Tijssen J, et al. Safety and efficacy outcomes of double vs. triple antithrombotic therapy in patients with atrial fibrillation following percutaneous coronary intervention: a systematic review and meta-analysis of non-vitamin K antagonist oral anticoagulant-based randomized clinical trials. Eur Heart J. 2019;40:3757-67.

20. Pisters R, Lane DA, Nieuwlaat R, et al. A novel user-friendly score (HAS-BLED) to assess 1-year risk of major bleeding in patients with atrial fibrillation: the Euro heart survey. Chest. 2010;138:1093-100.

21. Subherwal S, Bach R, Chen A, et al. Baseline risk of major bleeding in non-ST-segment elevation myocardial infarction: the CRUSADE (Can rapid risk stratification of unstable angina patients suppress adverse outcomes with early implementation of theACC/AHA guidelines) bleeding score. Circulation. 2009;119:1873-82.

22. Costa F, van Klaveren D, James S, et al. Derivation and validation of the predicting bleeding complications in patients 
undergoing stent implantation and subsequent dual antiplatelet therapy (PRECISE-DAPT) score: a pooled analysis of individual-patient datasets from clinical trials. Lancet. 2017;389:1025-34.

23. Yeh RW, Secemsky EA, Kereiakes DJ, et al. Development and validation of a prediction rule for benefit and harm of dual antiplatelet therapy beyond 1 year after percutaneous coronaryintervention. JAMA. 2016;315:1735-49.

24. Olesen JB, Lip GY, Hansen ML, et al. Validation of risk stratification schemes for predicting stroke and thromboembolism in patients with atrial fibrillation: nationwide cohort study. BMJ.2011;342:d124.

25. Steffel J, Verhamme P, Potpara TS, et al. The 2018 European heart rhythm association practical guide on the use of nonvitamin $\mathrm{K}$ antagonist oral anticoagulants in patients with atrial fibrillation. Eur Heart J. 2018;39:1330-93.
26. Lee K, Choi J, Boo KY, et al. Effectiveness and safety of offlabel dosing of non-vitamin K antagonist anticoagulant for atrial fibrillation in Asian patients. Sci Rep. 2020;10:1801.

27. Alexander JH, Lopes RD, James S, et al. Apixaban with antiplatelet therapy after acute coronary syndrome. N Engl JMed. 2011;365:699-708.

28. Mega JL, Braunwald E, Wiviott SD, et al. Rivaroxaban in patients with recent acute coronary syndrome. N Engl J Med. 2012;366:9-19.

29. Eikelboom JW, Connolly SJ, Bosch J, et al. Rivaroxaban with or without aspirin in stable cardiovascular disease. N Engl J Med. 2017;377:1319-30.

30. Yasuda S, Kaikita K, Akao M, et al. Antithrombotic therapy for atrial fibrillation with stable coronary disease. N Engl J Med. 2019;381:1103-13. 